\title{
Mobility \& Routing Control in Networks of Robots
}

\author{
Michael M. Zavlanos, Alejandro Ribeiro and George J. Pappas
}

\begin{abstract}
Most coordinated tasks performed by teams of mobile robots, require reliable communications between the members of the team. Therefore, task accomplishment requires that robots navigate their environment with their collective movement restricted to formations that guarantee integrity of the communication network. Maintaining this communication capability induces physical constraints on trajectories but also requires determination of communication variables like routes and transmitted powers. The development of theory and algorithms for joint control of mobility and communications is therefore necessary to facilitate efficient design of mobile autonomous systems. In this paper we address this challenge using a hybrid approach, where continuous motion controllers based on potential fields interact with discrete optimization of the communication variables to result is a muti-robot network that deploys itself to accomplish certain tasks, while ensuring integrity of communications. Our definition of network integrity differs from existing approaches in that it is not based on the topology of the network but on metrics that are of interest to the performance of communication between robots and possibly a fixed infrastructure. In this paper, integrity is defined as the ability of a network to support desired communication rates.
\end{abstract}

\section{INTRODUCTION}

Mobile robot networks have recently emerged as an inexpensive and robust way to address a wide variety of tasks ranging from exploration, surveillance and reconnaissance, to cooperative construction and manipulation. Efficient information exchange and coordination between members of the team are critical for successful completion of these tasks. In fact, recent results in distributed consensus have shown that multihop communication is necessary for convergence and performance of the algorithms under consideration [1]-[5].

Modeling communication in multi-robot systems has typically relied on constructs from graph theory, with disc and weight based models gaining the most popularity. This is consistent with early approaches to wireless networking that used disk models to abstract the physical layer [6], [7]. Nevertheless, the structural properties of graphs, such as topological connectivity, did not become a control objective until recently with the work of [8] on connectivity preserving rendezvous. Since then, a large amount of research has been targeted in this direction, and a wide range of applications and solution techniques have been proposed. Approaches can be classified into those that increase network connectivity [9]-[13] and less restrictive ones that allow links to be lost

This work is partially supported by the ONR HUNT MURI project

Michael M. Zavlanos is with the Dept. of Mechanical Engineering, Stevens Institute of Technology, Hoboken, NJ 07030, USA michael.zavlanos@stevens.edu. Alejandro Ribeiro and George J. Pappas are with the Dept. of Electrical and Systems Engineering, University of Pennsylvania, Philadelphia, PA 19104, USA \{aribeiro, pappasg\}@seas.upenn.edu.
[14]-[18]. Both centralized [9], [13], [16], [17] and distributed [10]-[12], [14], [18] solutions have been proposed, with the former typically based on semidefinite programming [9], [10], [16] or potential fields [13], and the latter relying mostly on switched and hybrid systems [11], [14], [18].

Although graphs provide a simple abstraction of interrobot communications, it has long being recognized that since links in a wireless network do not entail tangible connections, associating links with arcs on a graph can be somewhat arbitrary [19]. Since, even small differences in target strengths might result in dramatic differences in network topology [20], graph connectivity is necessary but not sufficient to guarantee communication integrity, which translates to the ability of a network to support desired communication rates. A simple, yet effective, modification is to use graph models that associate weights to links used to capture either the signal strength [21], or the packet error probability of the link [22], [23]. When using reliabilities as link metrics it is possible to model routing and scheduling problems as optimization problems that accept link reliabilities as inputs [24], [25].

The key idea proposed in this paper is to define network integrity as the set of routes and communication rates that describe optimal operating points of wireless networks. The use of optimization as a mathematical tool to analyze network protocols dates back to [26] and [27] and has been extensively used in wired [28], [29] and wireless networks [30]-[32]. General optimal wireless networking problems are defined to determine end-to-end user rates, routes, link capacities, and transmitted power, as well as frequency and power allocations [33]-[35]. While in general this leads to problems with substantial computational complexity recent results have shown that significant simplifications can be afforded by working in the dual domain [36]. The main contribution of this work is the use of optimal wireless network design to develop novel alternatives for mobility control. This leads to a hybrid control scheme where continuous motion control and discrete optimization of the communication variables jointly result in optimal network deployment, defined in terms of sustaining desired communication rates. We show that our approach ensures communication integrity of the mobile robot network both in theory and in simulation.

The rest of this paper is organized as follows. In Section II we define network integrity in terms of routing probabilities and communication rates. In Section III we develop the motion controllers for the robots and integrate them with optimization of the communication variables in a provably correct control scheme. Finally, in Section IV we illustrate our approach in various multi-robot tasks. 


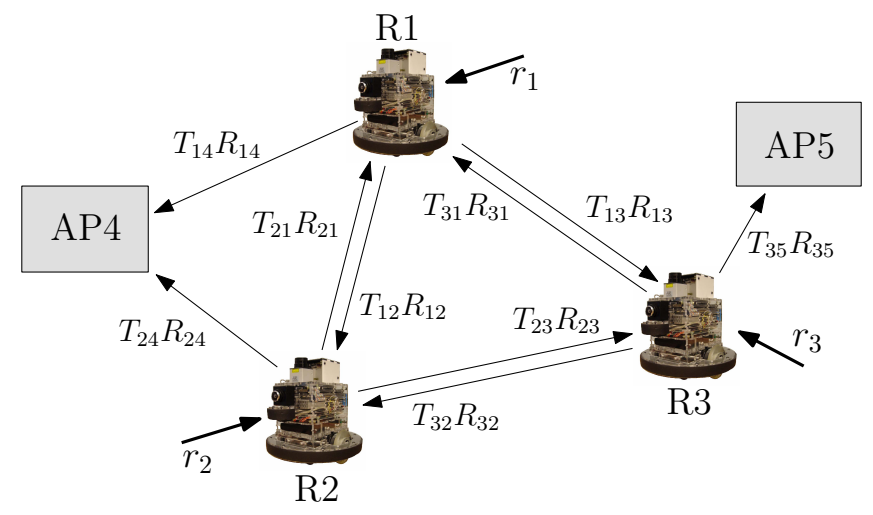

Fig. 1. Robotic network consisting of two access points (AP) and three robots (R). Shown are the packet rates $r_{i}$ generated by every robot as well as the rates $T_{i j} R\left(\mathbf{x}_{i}, \mathbf{x}_{j}\right)$ sent from robot $i$ and successfully decoded by robot $j$, where $T_{i j}$ is the probability that robot $i$ routes packets to robot $j$ and $R\left(\mathbf{x}_{i}, \mathbf{x}_{j}\right)$ is the reliability of the channel between robots $i$ and $j$.

\section{Problem Definition}

Consider a mobile network composed of $J$ robots and a fixed infrastructure with $K$ access points (APs). The robots move throughout an area of interest to accomplish an assigned task for which it is necessary to maintain reliable communications with the infrastructure. Due to, e.g., power constraints or an adverse propagation environment, robots collaborate to maintain a multihop network with the APs.

Let $\mathbf{x}_{j}$ for $j=1, \ldots, J$ denote the position of the robots and $\mathbf{x}_{j}$ for $j=J+1, \ldots, J+K$ the position of the APs. We model communication by a link reliability metric $R(\mathbf{x}, \mathbf{y})$ denoting the probability that a packet transmitted from a terminal located at position $\mathrm{x}$ is correctly decoded by a terminal at position $\mathbf{y}$. This function determines the probability

$$
R_{i j} \triangleq R\left(\mathbf{x}_{i}, \mathbf{x}_{j}\right)
$$

with which a packet transmitted by node $i$ is correctly decoded by node $j$. Node $j$ is a robot if $j \leq J$ or an AP otherwise. Furthermore, we denote by $r_{i}$ the average rate of information, i.e., packets per unit of time, aquired by every robot $i$. If robot $i$ can reach some of the APs, which is possible if the probability $R\left(\mathbf{x}_{i}, \mathbf{x}_{j}\right)$ is reasonably large for some $j \in\{J+1, \ldots, J+K\}$, packets are directly conveyed to the corresponding AP. Otherwise, packets are routed to another robot for subsequent transmission. In general, we model this process through the introduction of routing probabilities $T_{i j}$ denoting the probability with which robot $i$ selects node $j$, either a robot or an AP, as a destination of its transmitted packets. The proposed model is shown in Fig. 1.

Between the time of their generation or arrival from another robot and the time of their transmission packets are stored in a queue, as shown in Fig. 2. A packet leaves the queue at robot $i$ when it is transmitted to any other node $j$ and is successfully decoded by this intended next-hop. Since these two events are independent, the rate at which packets are sent from robot $i$ to node $j$ is $T_{i j} R\left(\mathbf{x}_{i}, \mathbf{x}_{j}\right)$. Thus, the

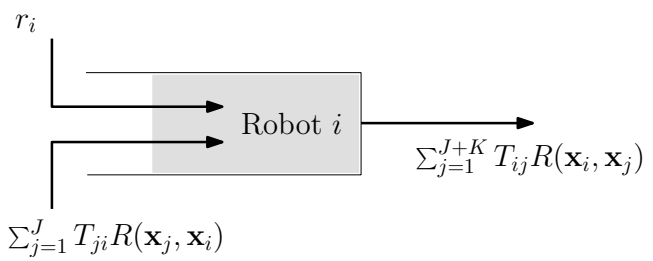

Fig. 2. Queue balance equations for robot $i$.

aggregate rate at which packets leave the $i$ th queue is

$$
r_{i}^{\text {out }}=\sum_{j=1}^{J+K} T_{i j} R\left(\mathbf{x}_{i}, \mathbf{x}_{j}\right) .
$$

Similarly, a packet enters the queue at robot $i$ coming from robot $j$, when robot $j$ selects $i$ as the next hop and $i$ correctly decodes the packet. This happens with probability $T_{j i} R\left(\mathbf{x}_{j}, \mathbf{x}_{i}\right)$. Considering that packets are also locally generated at a rate $r_{i}$, the rate at which packets arrive at the $i$ th queue is

$$
r_{i}^{\text {in }}=r_{i}+\sum_{j=1}^{J} T_{j i} R\left(\mathbf{x}_{j}, \mathbf{x}_{i}\right) .
$$

Note that the sum in (1) is up to $J+K$ because packets can be sent to another robot or an AP, whereas the sum in (2) is up to $J$ because packets are received from peer robots only (see also Fig. 1).

If the average rate at which packets arrive at the $i$ th queue is smaller than the average rate at which packets leave this queue, i.e., if $r_{i}^{\text {in }} \leq r_{i}^{\text {out }}$, the number of packets in queue remains bounded with probability one. This provides an almost sure guarantee that packets are eventually delivered to the AP as long as $r_{i}^{\text {in }} \leq r_{i}^{\text {out }}$. Thus, our interest is to determine routing probabilities $T_{i j}$ and rates $r_{i}$ that satisfy the inequality

$$
r_{i}+\sum_{j=1}^{J} T_{j i} R\left(\mathbf{x}_{j}, \mathbf{x}_{i}\right) \leq \sum_{j=1}^{J+K} T_{i j} R\left(\mathbf{x}_{i}, \mathbf{x}_{j}\right) .
$$

This gives rise to the notion of network integrity:

Definition 2.1: We define by network integrity the ability of all robots in a network to communicate with the infrastructure at a basal rate of $r_{i 0}$ packets per time unit.

Therefore, ensuring network integrity requires routing probabilities $T_{i j}$ and rates $r_{i}$ that satisfy (3) and also $r_{i} \geq r_{i 0}$ for all $i \in\{1, \ldots, K\}$. More generally, introduce a concave utility function $U_{i}\left(r_{i}\right)$ measuring the value associated with communication rate $r_{i}$ (see also Remark 2.2) and define the optimization problem

$$
\begin{aligned}
P=\max _{T_{i j}} & \sum_{i=1}^{J} U_{i}\left(r_{i}\right) \\
\text { s.t. } & r_{i}+\sum_{j=1}^{J} T_{j i} R\left(\mathbf{x}_{j}, \mathbf{x}_{i}\right) \leq \sum_{j=1}^{J+K} T_{i j} R\left(\mathbf{x}_{i}, \mathbf{x}_{j}\right), \\
& r_{i} \geq r_{i 0}, \quad \sum_{j=1}^{J} T_{i j} \leq 1,
\end{aligned}
$$


where the constraints are required for all $i \in\{1, \ldots, K\}$. To ensure network integrity for given robot positions $\mathbf{x}_{i}$, we need to find optimal routing probabilities $T_{i j}$ that solve the optimization problem in (4). This implies desired basal rates $r_{i 0}$ for all terminals, while assigning the remaining resources in a manner that is optimal in terms of the utilities $U_{i}\left(r_{i}\right)$. For fixed position $\mathbf{x}_{i}$, the reliabilities $R\left(\mathbf{x}_{i}, \mathbf{x}_{j}\right)$ are fixed and the problem in (4) attains a simple convex form. However, this is not the case for mobile robots that are supposed to move to accomplish their assigned task. In particular, we consider single integrator robots whose positions $\mathbf{x}_{i}$ react to control inputs $\mathbf{u}_{i}$ according to the first order differential equation

$$
\dot{\mathbf{x}}_{i}=\mathbf{u}_{i}, \quad i=1, \ldots, J .
$$

The problem of joint motion and routing control for network integrity can now be defined as follows:

Problem 1: Determine motion controllers $\mathbf{u}_{i}$ and routes $\left\{T_{i j}\right\}_{j=1}^{J+K}$ for all robots $i$ that ensure task completion as well as communication rates $r_{i}$ that exceed $r_{i 0}$ at all times.

Remark 2.2 (Utility functions): The utilities $U_{i}\left(r_{i}\right)$ in problem (4) are metrics used to compare different operating points of the wireless network. Thinking of nodes as economic agents and of utilities $U_{i}\left(r_{i}\right)$ as the value of rate $r_{i}$, the network's objective is to maximize the social value $\sum_{i} U_{i}\left(r_{i}\right)$. Utilities employed in practice are typically linear $U_{i}\left(r_{i}\right)=w_{i} r_{i}$ or logarithmic $U_{i}\left(r_{i}\right)=\log \left(r_{i}\right)$. Linear utilities yield larger rates, while logarithmic utilities yield fairer operating points because they penalize small rates $r_{i}$. Utility functions can be used to implement congestion control, by determining rates that are as good as possible while preventing the appearance of bottlenecks. Alternatively, they can be used for power management of the network.

\section{Mobility \& Routing CONTROL}

Since mobility introduces nonlinearities in (4), we propose a parallel control scheme, where motion control and control of communication variables are performed simultaneously in different time scales. Integration of the two gives rise to a hybrid control scheme, where the discrete-time routing probabilities become the switching signal in the continuoustime motion controllers. To simplify the problem a little, we first consider task completion as a secondary objective, subsidiary to communication integrity. While this implies that robots may not be able to complete their assigned tasks, it is consistent with the idea that basal rates $r_{i 0}$ are critical for task completion.

Let $\phi_{i}: \mathbb{R}^{d J} \rightarrow \mathbb{R}_{+}$denote an artificial potential function associated with every robot $i$, such that ${ }^{1}$

$$
\phi_{i} \triangleq \phi_{i, 1}+\phi_{i, 2}+\phi_{i, c}+\phi_{i, t},
$$

where $d>0$ denotes the dimension of the free space. The potentials $\phi_{i, t}$ and $\phi_{i, c}$ capture the task that robot $i$ needs to complete and collision avoidance with close-by robots, respectively, while $\phi_{i, 1}$ and $\phi_{i, 2}$ are barrier potentials

\footnotetext{
${ }^{1}$ We denote by $\mathbb{R}_{+}$the set $[0, \infty)$.
}

associated with the first two constraints of the optimization problem in (4). In particular we define the potential

$$
\phi_{i, 1} \triangleq\left[\left(\sum_{j=1}^{J+K} T_{i j} R_{i j}\right)^{2}-\left(\sum_{j=1}^{J} T_{j i} R_{j i}+r_{i}\right)^{2}\right]^{-1}
$$

that is associated with the first constrain in (4) and ensures internal consistency of routing variables $T_{i j}$ and rates $r_{i}$, and the barrier potential

$$
\phi_{i, 2} \triangleq \frac{1}{r_{i}^{2}-r_{i 0}^{2}},
$$

that captures the second constrain in (4) and ensures compliance with the demanded basal rates $r_{i 0}$. Collision avoidance is guaranteed by the potential

$$
\phi_{i, c} \triangleq \sum_{j \neq i} \frac{1}{\left\|\mathbf{x}_{i}-\mathbf{x}_{j}\right\|_{2}^{2}},
$$

while the task potentials $\phi_{i, t}$ can be designed to model a variety of tasks. An example problem is that of a lead robot required to service a target location $\mathbf{x}_{i, t}$, as discussed in Section IV. Then, the set of controllers

$$
\begin{aligned}
\dot{\mathbf{x}}_{i} & =-\nabla_{\mathbf{x}_{i}} \phi_{i} \\
\dot{r}_{i} & =-\nabla_{r_{i}} \phi_{i}
\end{aligned}
$$

for all $i=1, \ldots, J$, simultaneously enforces the constraints in (4) and drives the robots to accomplish their tasks. In other words, (10) employs robot motion to control the channel reliabilities $R_{i j}=R\left(\mathbf{x}_{i}, \mathbf{x}_{j}\right)$ so that for fixed routes $T_{i j}$, communication integrity with the infrastructure is maintained. Composition with periodic updating of the routes $T_{i j}$ by the solution of problem (4), results in a hybrid closed loop system for which we show the following result:

Proposition 3.1 (Network Integrity): The closed loop system (4) - (10) guarantees that all robots can communicate with the infrastructure at a basal rate of $r_{i 0}$ packets per unit time. Moreover, the robot velocities are bounded and collisions between robots are avoided.

Proof: Consider the potential function $\phi: \mathbb{R}^{d J} \rightarrow \mathbb{R}_{+}$ such that

$$
\phi=\sum_{i=1}^{J} \phi_{i}
$$

where $d$ denotes the workspace dimension and for any $c>0$ define the set $\Omega_{c} \triangleq\left\{\mathrm{x} \in \mathbb{R}^{d J} \mid \phi \leq c\right\}$. Observe that

$$
\Omega_{c} \subseteq \cap_{i=1}^{J}\left(\phi_{i, 1}^{-1}([0, c]) \cap \phi_{i, 2}^{-1}([0, c]) \cap \phi_{i, t}^{-1}([0, c])\right) \triangleq \Omega .
$$

The sets $\phi_{i, t}^{-1}([0, c])$ are closed by continuity of the potentials $\phi_{i, t}$ in $\mathbb{R}^{d}$. They are also bounded; to see this, suppose that there exists an $i$ for which $\phi_{i, t}^{-1}([0, c])$ is unbounded. Then, for any choice of $N>0$, there exists an $\mathbf{x}_{i} \in$ $\phi_{i, t}^{-1}([0, c])$ such that $\left\|\mathbf{x}_{i}\right\|_{2}>N$. Allowing $N \rightarrow \infty$ and given that $\lim _{\left\|\mathbf{x}_{i}\right\|_{2} \rightarrow \infty} \phi_{i, t}\left(\mathbf{x}_{i}\right)=\infty$, it follows that for any $M>0$, there is an $N>0$ such that $\phi_{i, t}\left(\mathbf{x}_{i}\right)>M$. If we pick 


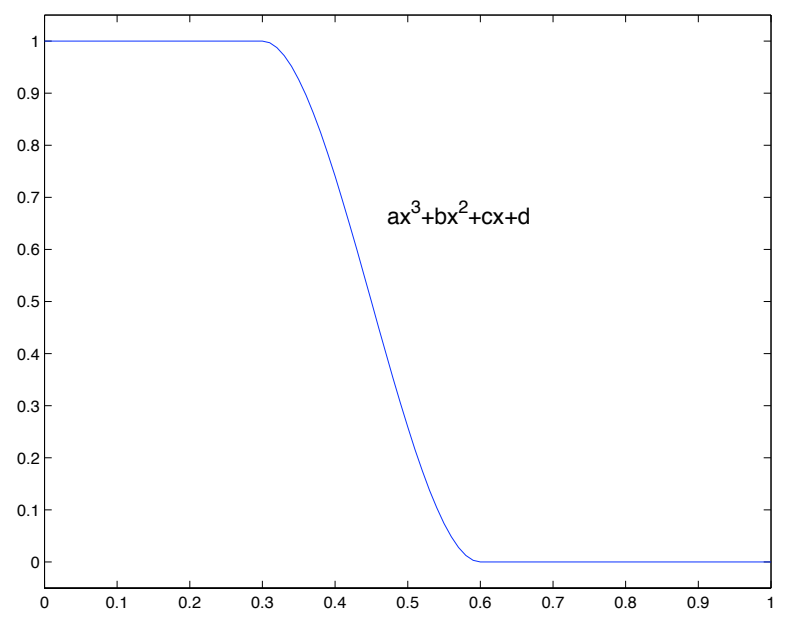

Fig. 3. Channel reliability $R_{i j}=R\left(\mathbf{x}_{i}, \mathbf{x}_{j}\right)$ for $l=.3$ and $u=.6$ and the scenario illustrated in Fig. 4.

$M>c$ we reach a contradiction, since by definition $\mathbf{x}_{i} \in$ $\phi_{i, t}^{-1}([0, c])=\left\{\mathbf{x}_{i} \mid \phi_{i, t}\left(\mathbf{x}_{i}\right) \leq c\right\}$. Thus, all sets $\phi_{i, t}^{-1}([0, c])$ are bounded and hence, compact. Similarly, we can show that the sets $\phi_{i, 1}^{-1}([0, c])$ and $\phi_{i, 2}^{-1}([0, c])$ are also compact. Therefore, the set $\Omega$ is compact as a finite intersection of compact sets. It follows that $\Omega_{c}$ is also compact, as a closed subset of a compact set.

The time derivative of $\phi$ in the set $\Omega_{c}$ is

$$
\dot{\phi}=-\sum_{i=1}^{J}\left(\left\|\nabla_{\mathbf{x}_{i}} \phi_{i}\right\|_{2}^{2}+\left\|\nabla_{r_{i}} \phi_{i}\right\|_{2}^{2}\right) \leq 0
$$

which implies that the level sets $\Omega_{c}$ of $\phi$ are also positively invariant. The invariance of $\Omega_{c}$ implies that the constraints in problem (4) are satisfied and, hence, communication integrity is guaranteed between consecutive solutions of problem (4). Communication integrity for all time follows from the observation that feasibility is maintained by the solution of problem (4). A similar argument shows collision avoidance. On the other hand, compactness and positive invariance of $\Omega_{c}$ implies that $\mathbf{x} \in \mathbb{R}^{d J}$ remains bounded for all time $t$ between any two consecutive solutions of problem (4). Since $\phi$ is twice differentiable inside $\mathbb{R}^{d J}$, the right-hand-side of the closed loop system defined by equations (10) is locally Lipschitz, which implies that $\dot{\mathbf{x}}$ is bounded. Hence, all agent velocities are bounded.

As discussed above, we consider task completion a secondary objective. The robots will do their best towards this end, until they are trapped at local minima of (6). Note also that although motion control (10) is distributed as nonadjacent robot links, i.e., links $R_{j k}$ with $j, k \neq i$, are not included in the potentials (7) of robot $i$, control of the routing probabilities $T_{i j}$ in problem (4) is centralized and, therefore, composition of the two controllers is centralized as well.

\section{INTEGRATION WITH ROBOT TASKS}

In this section we illustrate our approach in different scenarios where communication integrity of the robot net- work needs to be preserved. For this, we employ channel reliabilities that satisfy

$$
R_{i j} \triangleq \begin{cases}a\left\|\mathbf{x}_{i j}\right\|_{2}^{3}+b\left\|\mathbf{x}_{i j}\right\|_{2}^{2}+c\left\|\mathbf{x}_{i j}\right\|_{2}+d, & \text { if } l \leq \mathbf{x}_{i j} \leq u \\ 0, & \text { otherwise }\end{cases}
$$

where $\mathbf{x}_{i j} \triangleq \mathbf{x}_{i}-\mathbf{x}_{j}$, the constants $0<l<u$ are lower and upper bounds on the inter-robot distances, respectively, and

$a \triangleq \frac{-2}{(l-u)^{3}}, b \triangleq \frac{3(l+u)}{(l-u)^{3}}, c \triangleq \frac{-6 l u}{(l-u)^{3}}, d \triangleq \frac{3 l u^{2}-u^{3}}{(l-u)^{3}}$,

such that $R_{i j}=R\left(\mathbf{x}_{i}, \mathbf{x}_{j}\right)$ is a twice differentiable function ranging from 0 to 1 (Fig. 3). This is a polynomial fitting of experimental curves found in the literature, e.g., [37].

We consider a scenario where reliable communications need to be established between $K=2$ access points (APs) and a single service point (SP) in $\mathbb{R}^{2}$. This task is implemented by a team of $J=8$ robots, with one being the leader that is responsible for serving the SP and the other robots relaying information back to the APs. The task potentials $\phi_{i, t}$ are identically zero for all robots except for the leader robot for which

$$
\phi_{i, t} \triangleq \frac{1}{2}\left\|\mathbf{x}_{i}-\mathbf{x}_{i, t}\right\|_{2}^{2}
$$

where $\mathbf{x}_{i, t}$ denotes the location of the service point. Finally, the rates $r_{i}$ are identically zero for all robots except for the leader for which $r_{i}=.8$. This formulation is consistent with the classification of robots into relay robots and leaders, since leaders collect measurements and generate data, while relay robots relay this information back to the access points. In this scenario, the utilities $U_{i}\left(r_{i}\right)$ of the robots are all chosen identically zero.

The task under consideration is illustrated in Fig. 4. We observe that the leader robot moves towards the SP under the influence of its task potential $\phi_{i, t}$ and forces the network to stretch in order to achieve reliable communications between the SP and the APs. Our algorithm tends to maintain high channel reliabilities as shown in Fig. 5(a), with the lowest ones corresponding to the leader robot. This is expected, since the leader is the input to the network that forces it to stretch and decrease the link reliabilities. On the other hand, communication integrity is identified by the rate at which packets are sent out from robot $i$ and are successfully decoded by robot or AP $j$. We capture link quality by the product $T_{i j} R_{i j}$ and denote weak links corresponding to $.1<T_{i j} R_{i j}<.3$ by dashed lines, and high quality links corresponding to $T_{i j} R_{i j}>.3$ by solid lines (Fig. 4). Links weaker than .1 are not shown in this simulation. ${ }^{2}$ Note that most packets are not necessarily routed through the most reliable links (Figs. 5(b) and 5(c)). Moreover, the robots that form a chain behind the leader (Fig. 4(f)) route all the packets to a single adjacent robot in the chain, which explains the subset of high rates in Figs. 5(b) and 5(c). The remaining

\footnotetext{
${ }^{2}$ The thresholds .1 and .3 were chosen arbitrarily for illustration only.
} 

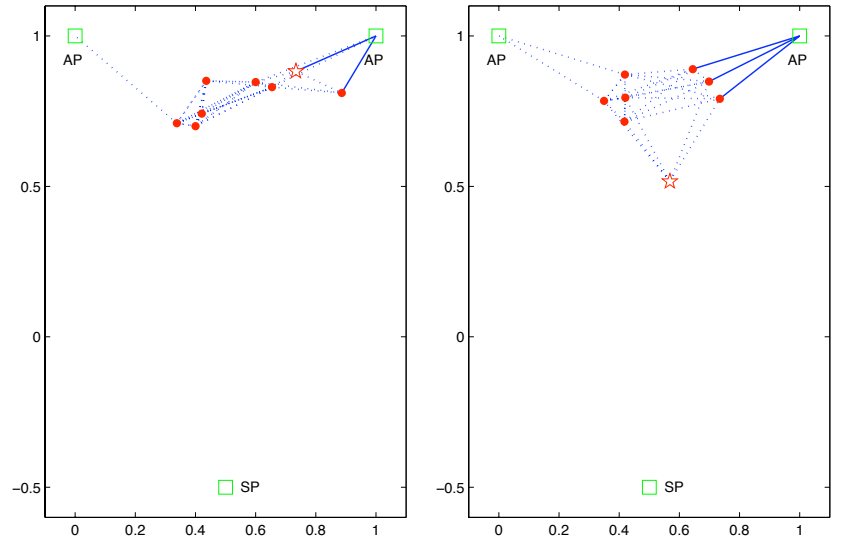

(a)

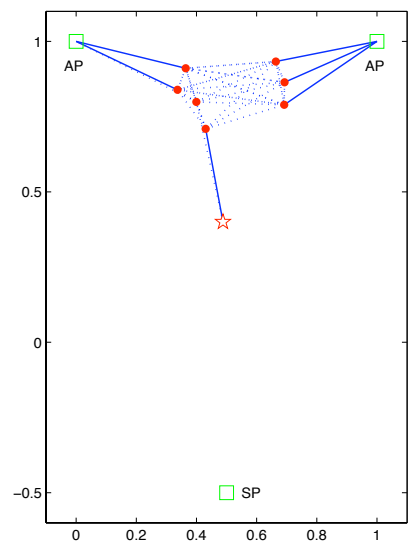

(c)

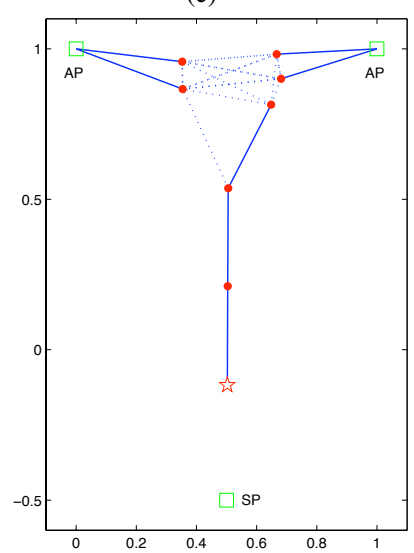

(e) (b)

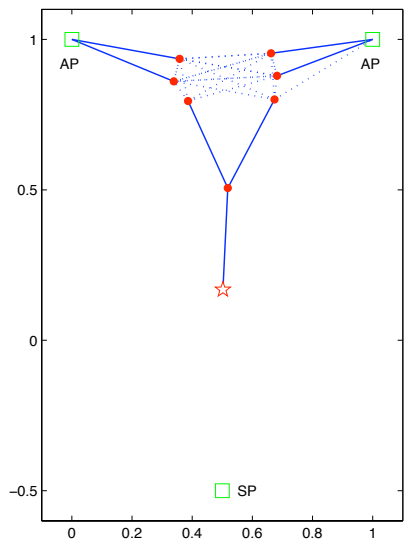

(d)

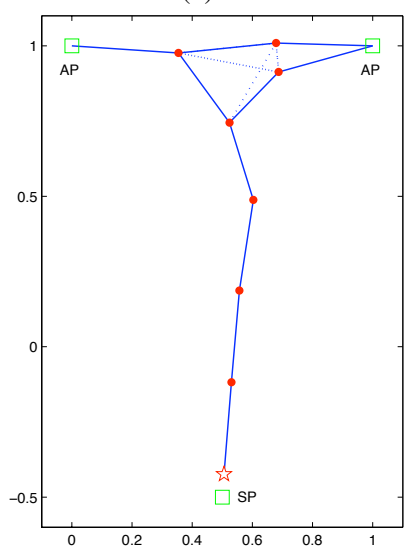

(f)

Fig. 4. Establishing reliable communications in a network consisting of $J=8$ robots and $K=2$ access points (APs). The leader's (star) task is to establish communication between a service point (SP) and the APs. Communication links are solid or dashed depending on their quality $T_{i j} R_{i j}$, with solid ones indicating higher quality. Packet flow is towards the APs (not shown in the figure).

robots split the packets between the two access points, hence the remaining lower rates.

\section{CONCLUSiOnS}

In this paper we considered the problem of ensuring communication integrity in networks of mobile robots. Unlike

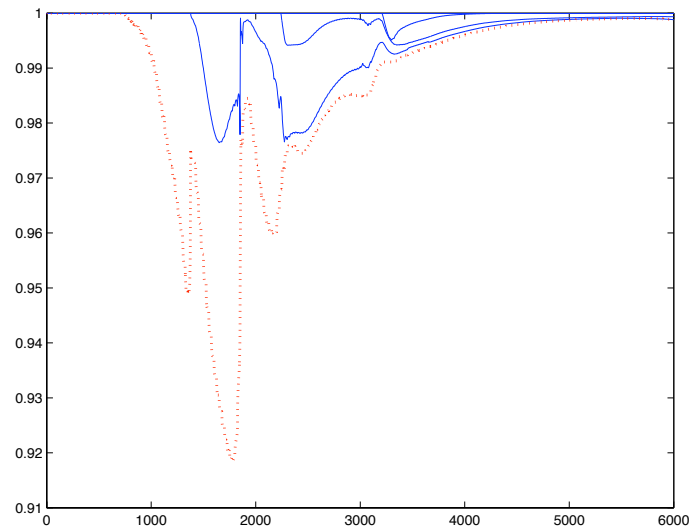

(a)

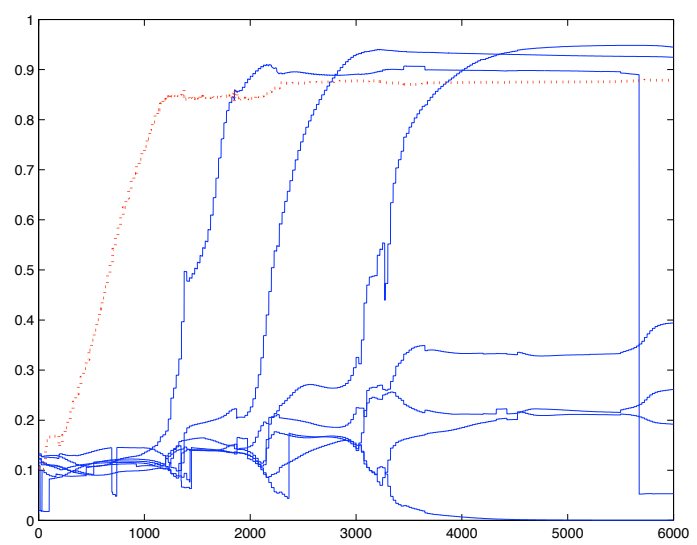

(b)

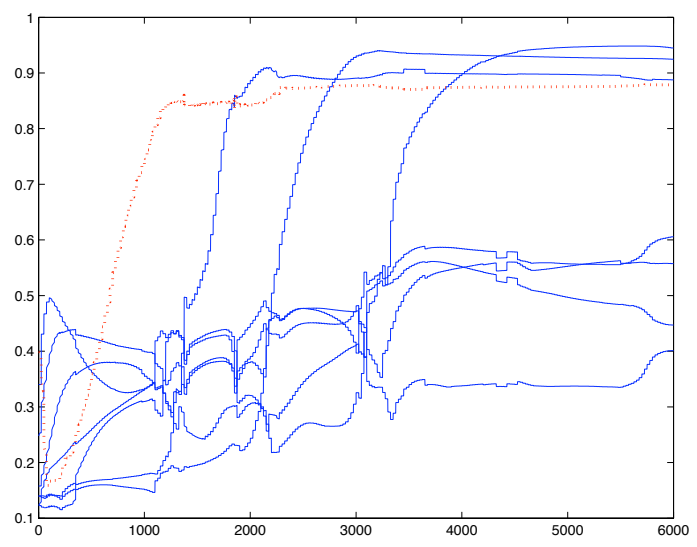

(c)

Fig. 5. Performance metrics for the scenario shown in Fig. 4. Solid lines correspond to the relay robots and dotted lines to the leader. Fig. 5(a) plots the reliability $R_{i j}$ of the most reliable link for every robot $i$ as a function of time. As expected, the leader robot has the lowest reliability over time, since it is the driving force that stretches the network. Fig. 5(b) contains the plots of the rates $T_{i j} R_{i j}$ associated with the most reliable links, while Fig. 5(c) plots the maximum rates for every robot. Observe that the maximum rates do not necessarily correspond to the most reliable links.

the recently popular notions of graph connectivity that are only necessary but not sufficient for reliable communications, network integrity is itself defined as the ability of a network to support desired communication rates. Our approach re- 
lied on introducing weights on the communication links to capture channel reliabilities, which then allowed to model routing by means of optimization problems that accept link reliabilities as inputs. The key idea proposed in this work was to join control of mobility and communications in a hybrid scheme with the discrete-time routing variables being the switching signal in the continuous-time motion controllers. We showed correctness of our approach and discussed various tasks that can be achieved while ensuring integrity of the communication network. We believe that this work points to a new direction in systems and control theory on the interface with wireless networking.

\section{REFERENCES}

[1] J. A. Fax and R. M. Murray, "Information flow and cooperative control of vehicle formations," IEEE Transactions on Automatic Control, vol. 49, no. 9, pp. 1465-1476, Sptember 2004.

[2] A. Jadbabaie, J. Lin, and A. S. Morse, "Coordination of groups of mobile autonomous agents using nearest neighbor rules," IEEE Transactions on Automatic Control, vol. 48, no. 6, pp. 988-1001, June 2003.

[3] L. Moreau, "Stability of multi-agent systems with time-dependent communication links," IEEE Transactions on Automatic Control, vol. 50, no. 2, pp. 169-182, February 2005.

[4] W. Ren and R. W. Beard, "Consensus seeking in multi-agent systems under dynamically changing interaction topologies," IEEE Transactions on Automatic Control, vol. 50, no. 5, pp. 655-661, May 2005.

[5] R. Olfati-Saber, J. A. Fax, and R. M. Murray, "Consensus and cooperation in networked multi-agent systems," Proc. of the IEEE, vol. 95, no. 1, pp. 215-233, January 2007.

[6] I. Stojmenovic, A. Nayak, and J. Kuruvila, "Design guidelines for routing protocols in ad hoc and sensor networks with a realistic physical layer," IEEE Communications Magazine, vol. 43, pp. 101106, March 2005.

[7] A. Neskovic, N. Neskovic, and G. Paunovic, "Modern approaches in modeling of mobile radio systems propagation environment," IEEE Communications Surveys, vol. 3, no. 3, pp. 1-12, 2000.

[8] H. Ando, Y. Oasa, I. Suzuki, and M. Yamashita, "Distributed memoryless point convergence algorithm for mobile robots with limited visibility," IEEE Transactions on Robotics and Automation, vol. 15, no. 5, pp. 818-828, October 1999.

[9] Y. Kim and M. Mesbahi, "On maximizing the second smallest eigenvalue of a state-dependent graph laplacian," IEEE Transactions on Automatic Control, vol. 51, no. 1, pp. 116-120, January 2006.

[10] M. C. DeGennaro and A. Jadbabaie, "Decentralized control of connectivity for multi-agent systems," in Proc. 45th IEEE Conference on Decision and Control, San Diego, CA, December 2006, pp. 36283633.

[11] M. Ji and M. Egerstedt, "Coordination control of multi-agent systems while preserving connectedness," IEEE Transactions on Robotics, vol. 23, no. 4, pp. 693-703, August 2007.

[12] G. Notarstefano, K. Savla, F. Bullo, and A. Jadbabaie, "Maintaining limited-range connectivity among second-order agents," in Proc. 2006 American Control Conference, Minneapolis, MN, June 2006, pp. 2124-2129.

[13] M. M. Zavlanos and G. J. Pappas, "Potential fields for maintaining connectivity of mobile networks," IEEE Transactions on Robotics, vol. 23, no. 4, pp. 812-816, August 2007.

[14] M. Schuresko and J. Cortes, "Distributed motion constraints for algebraic connectivity of robotic networks," Journal of Intelligent and Robotic Systems, vol. 56, no. 1-2, pp. 99-126, September 2009.

[15] D. P. Spanos and R. M. Murray, "Motion planning with wireless network constraints," in Proc. 2005 American Control Conference, Portland, OR, June 2005, pp. 87-92.

[16] E. Stump, A. Jadbabaie, and V. Kumar, "Connectivity management in mobile robot teams," in Proc. IEEE International Conference on Robotics and Automation, Pasadena, CA, May 2008, pp. 1525-1530.

[17] M. M. Zavlanos and G. J. Pappas, "Controlling connectivity of dynamic graphs," in Proc. 44th IEEE Conference on Decision and Control and European Control Conference, Seville, Spain, December 2005, pp. 6388-6393.
[18] — "Distributed connectivity control of mobile networks," IEEE Transactions on Robotics, vol. 24, no. 6, pp. 1416-1428, December 2008.

[19] A. Ephremides, "Energy concerns in wireless networks," IEEE Transactions on Wireless Communications, vol. 9, no. 4, pp. 48-59, August 2002.

[20] H. Lundgren, E. Nordstrom, and C. Tschudin, "The gray zone problem in ieee $802.11 \mathrm{~b}$ based ad hoc networks," ACM SIGMOBILE Mobile Computing and Communications Review, vol. 6, no. 3, pp. 104-105, July 2002.

[21] R. Zhang and M. Alouini, "Channel-aware inter-cluster routing protocol for wireless ad-hoc networks," in Proc. of the 6th International Symposium on Communication Theory and Applications, Ambleside, UK, July 2001, pp. 46-51.

[22] D. DeCouto, D. Aguayo, J. Bicket, and R. Morris, "A high-throughput path metric for multihop wireless routing," in Proc. ofInternational ACM Conference on Mobile Computing and Networking, San Diego, CA, September 2006, pp. 134-146.

[23] S. Biswas and R. Morris, "Exor: opportunistic multihop routing for wireless networks," ACM SIGCOMM Computer Communication Review, vol. 35, no. 4, pp. 133-144, October 2005.

[24] A. Ribeiro, Z.-Q. Luo, N. D. Sidiropoulos, and G. B. Giannakis, "Modelling and optimization of stochastic routing for wireless multihop networks," in Proc. 26th Annual Joint Conference of the IEEE Computer and Communications Societies (INFOCOM), Anchorage, Alaska, May 2007, pp. 1748-1756.

[25] A. Ribeiro, N. D. Sidiropoulos, and G. B. Giannakis, "Optimal distributed stochastic routing algorithms for wireless multihop networks," IEEE Transactions on Wireless Communications, vol. 7, no. 11, pp. 4261-4272, November 2008

[26] F. P. Kelly, A. Maulloo, and D. Tan, "Rate control for communication networks: Shadow prices, proportional fairness and stability," Journal of the Operational Research Society, vol. 49, no. 3, pp. 237-252, 1998.

[27] S. H. Low and D. E. Lapsley, "Optimization flow control - i: basic algorithm and convergence," IEEE/ACM Transactions on Networking, vol. 7, no. 6, pp. 861-874, December 1999.

[28] S. H. Low, F. Paganini, and J. C. Doyle, "Internet congestion control," IEEE Control Systems Magazine, vol. 22, pp. 28-43, February 2002.

[29] R. Srikant, The Mathematics of Internet Congestion Control (1st Edition). Boston, MA: Birkhauser, 2004.

[30] L. Chen, S. H. Low, M. Chiang, and J. C. Doyle, "Cross-layer congestion control, routing and scheduling design in ad hoc wireless networks," in Proc. 24th Annual Joint Conference of the IEEE Computer and Communications Societies (INFOCOM), Barcelona, Spain, April 2005, pp. 1-13.

[31] X. Lin, N. B. Shroff, and R. Srikant, "A tutorial on cross-layer optimization in wireless networks," IEEE Journal on Selected Areas in Communications, vol. 24, no. 8, pp. 1452-1463, August 2006.

[32] Y. Yi and S. Shakkottai, "Hop-by-hop congestion control over a wireless multi-hop network," IEEE/ACM Transactions on Networking, vol. 15, no. 1, pp. 1548-1559, February 2007.

[33] M. J. Neely, "Energy optimal control for time-varying wireless networks," IEEE Transactions on Information Theory, vol. 52, no. 7, pp. 2915-2934, July 2006.

[34] M. Chiang, "Balancing transport and physical layers in wireless multihop networks: jointly optimal congestion control and power control," IEEE Journal on Selected Areas in Communications, vol. 23, no. 1, pp. 104-116, January 2005.

[35] L. Xiao, M. Johansson, and S. Boyd, "Simultaneous routing and resource allocation via dual decomposition," IEEE Transactions on Communications, vol. 52, no. 7, pp. 1136-1144, July 2004.

[36] A. Ribeiro and G. B. Giannakis, "Optimal fdma over wireless fading mobile ad-hoc networks," in Proc. of the IEEE International Conference on Acoustics, Speech, and Signal Processing (ICASSP), Las Vegas, NV, March 2008, pp. 2765-2768.

[37] D. Aguayo, J. Bicket, S. Biswas, G. Judd, and R. Morris, "Linklevel measurements from an $802.11 \mathrm{~b}$ mesh network," ACM SIGCOMM Computer Communication Review, vol. 34, no. 4, pp. 121-132, October 2004. 